\title{
Development of Miniaturized Hollow-fiber Assisted Liquid-phase Microextraction with in situ Acyl Derivatization Followed by GC-MS for the Determination of Benzophenones in Human Urine Samples
}

\author{
Rie Ito, ${ }^{* \dagger}$ Migaku KawaguchI,** Youji KoganeI,* Hidehiro Honda,* Noriya OKAnOuchI,* \\ Norihiro SAKUI,*** Koichi SAITo, ${ }^{*}$ and Hiroyuki NAKAZAWA* \\ *Department of Analytical Chemistry, Faculty of Pharmaceutical Sciences, Hoshi University, \\ 2-4-41 Ebara, Shinagawa, Tokyo 142-8501, Japan \\ **Bio-Medical Standard Section, National Metrology Institute of Japan (NMIJ), \\ National Institute of Advanced Industrial Science and Technology (AIST), \\ Tsukuba Central 3, 1-1-1 Umezono, Tsukuba, Ibaraki 305-8563, Japan \\ ***Agilent Technologies, Hachioji Site, 9-1 Takakura, Hachioji, Tokyo 192-0033, Japan
}

\begin{abstract}
A simple and highly sensitive method that involves miniaturized hollow fiber assisted liquid-phase microextraction (HF-LPME) with in situ acyl derivatization and GC-MS was developed for the determination of benzophenone (BP) and related compounds in human urine samples. The limits of detection $(S / N=3)$ and quantification $(S / N>10)$ of $\mathrm{BPs}$ in human urine samples are 0.01 to 0.05 and 0.05 to $0.2 \mathrm{ng} \mathrm{ml}^{-1}$, respectively. The average recoveries of BPs $(n=5)$ in human urine samples spiked with 10 and $50 \mathrm{ng} \mathrm{ml}^{-1}$ BPs are 93.1 to $106.7 \%$ (RSD: 1.5 to $8.4 \%$ ) and 96.3 to $101.5 \%$ (RSD: 3.0 to $7.7 \%$ ), respectively. When the proposed method was applied to human urine samples, BPs were detected at the sub ng ml-1 level.
\end{abstract}

(Received February 20, 2009; Accepted May 21, 2009; Published August 10, 2009)

\section{Introduction}

Benzophenone (diphenylmethanone; BP) and related compounds are widely used as chemical sunscreens. They absorb damaging UV rays to decrease the radiation dose, and are widely used in cosmetic products. Benzophenones (BPs) demonstrate maximum absorption at wavelengths of 288 to 290 and $325 \mathrm{~nm}$, and have the property of absorbing wavelengths of 200 to $400 \mathrm{~nm}$. Consequently, BPs are able to absorb UV light that is harmful to the human body in the form of UVA (320 to $400 \mathrm{~nm}$ ) and UVB (290 to $320 \mathrm{~nm}$ ), and have been reported to be effective in preventing skin disorders and skin cancer. ${ }^{1}$ However, BPs are also suspected to cause pruritus and contact allergies, ${ }^{2}$ and to disrupt the endocrine system by exerting estrogenic and anti-androgenic action. ${ }^{3-5}$ Recently, the migration of BPs from multilayer plastic-paper materials intended for food packaging and the contamination of food sample with BPs have been reported. ${ }^{6,7}$ Due to the widespread usage of BPs, healthy humans may be exposed to BPs via a variety of daily activities. Therefore, the assessment of human exposure to BPs is an important task.

It has been reported that when a human ingests $\mathrm{BP}$, it is excreted in urine as a metabolite, such as benzhydrol (BP-OH) glucuronide. $^{8}$ Thus, it is thought that human exposure can be evaluated by measuring these compounds in human urine samples. However, as the concentration of BPs in human urine

† To whom correspondence should be addressed.

E-mail: rie-ito@hoshi.ac.jp is low, a method with high sensitivity and high accuracy is required.

The determination of BPs in human urine samples has been accomplished with LC with diode array detection, ${ }^{9}$ tandem mass spectrometry (MS-MS) ${ }^{10,11}$ and GC-MS. ${ }^{12}$ However, these techniques require that such pretreatment procedures as liquid-liquid extraction (LLE) ${ }^{13,14}$ and solid phase extraction (SPE) ${ }^{11,15}$ be performed prior to use, and consume considerable time and labor. As for the time and labor needed for analysis, solid-phase microextraction (SPME) has been successfully used for the determination of BPs in human urine samples. ${ }^{12}$ However, because the limit of detection (LOD) of 2-hydroxy-4methoxybenzophenone (BP-3) is $5 \mathrm{ng} \mathrm{ml}^{-1}$, the sensitivity of the above SPME method remains low. Previously, we reported on a stir bar sorptive extraction (SBSE) method that uses a stir bar coated with polydimethylsiloxane (PDMS) for the determination of BPs in water samples ${ }^{16,17}$ and human urine samples. ${ }^{18}$ The SBSE method required not only a PDMS-coated stir bar, but also a thermal desorption (TD)-GC-MS system. The TD system had a high running cost because liquid nitrogen was used. To improve the cost performance, liquid-phase microextraction (LPME), solvent microextraction (SME) and a single-drop microextraction (SDME) techniques have been developed. ${ }^{19-21}$ LPME consists of extracting and concentrating a target compound with an extremely small amount of extraction solvent using a commercially available microsyringe. Highly sensitive trace analysis is subsequently performed by injecting the extract directly into a GC-MS system using the same microsyringe as that used to collect the extract. The main advantages of these techniques are good cost performance and a wide application 
range that includes polyaromatic hydrocarbons, ${ }^{22-24}$ polychlorinated biphenyls (PCBs), ${ }^{25,26}$ pesticides, ${ }^{24,26-29}$ and organotin compounds. ${ }^{30}$ Because of the interfacial activity of urine samples, it is difficult to retain a single droplet on the microsyringe needle tip. ${ }^{31}$ Therefore, we have developed miniatulized hollow fiber (HF) assisted LPME for BPs analysis. ${ }^{32}$ In that study, ${ }^{32}$ five kinds of BPs (BP, BP-OH, 2OH-BP, BP-3, and BP-10) could be analyzed without derivatization. Because of their high polarity and low volatility, phenol compounds including BPs are poorly separated by GC. Derivatization has yielded sharper peaks, and hence better separation of and higher sensitivity for the phenols. However, the derivatization procedure is tedious and time consuming. In order to avoid this problem, in situ derivatization was developed. Moreover, with an in situ derivatization step, an even wider variety of BP-related compounds could be analyzed to reveal the extent of exposure to BPs.

The aim of this study was to develop an analytical method for the trace analysis of eight kinds of BPs in human urine samples, which employs miniaturized HF-LPME with in situ derivatization and GC-MS. The HF-LPME method was performed in conventional $2 \mathrm{ml}$ vials for miniaturization. To consider a further application to valuable samples, such as serum, we performed a miniaturized HF-LPME method.

\section{Experimental}

\section{Materials and reagents}

Acetonitrile and methanol were purchased from Wako Pure Chemical (Osaka, Japan). BP and deuterium-labeled benzophenone- $d_{10} \quad\left(\mathrm{BP}-d_{10}\right)$ as surrogate compounds were purchased from Kanto Chemical (Tokyo, Japan). 2,4-Dihydroxybenzophenone (BP-1) and BP-3 were obtained from Sigma-Aldrich (St. Louis, MO). 2-Hydroxy-4-methoxy4'-methylbenzophenone (BP-10) was obtained from Lancaster Synthesis (Morecambe, England). Benzhydrol (BP-OH), 2-hydroxybenzophenone (2OH-BP), 3-hydroxybenzophenone (3OH-BP), and 4-hydroxybenzophenone (4OH-BP) were purchased from Wako Pure Chemical. E. coli $\beta$-glucuronidase (25000 $\left.0.4 \mathrm{ml}^{-1}\right)$ and $H$. pomatia sulfatase $\left(3650 \mathrm{U} \mathrm{ml}^{-1}\right)$ were purchased from Sigma-Aldrich. Prior to use, $\beta$-glucuronidase was added to $0.1 \mathrm{M}$ ammonium acetate to make a total concentration of $10000 \mathrm{U} \mathrm{ml}^{-1}$. Other reagents and solvents of pesticide or analytical grade were purchased from Wako Pure Chemical (Osaka, Japan). The water purification system was a Milli-Q gradient A 10 with an EDS polisher (Millipore, Bedford, MA).

Concentrated solutions $\left(1.0 \mathrm{mg} \mathrm{m}^{-1}\right.$ in methanol) of $\mathrm{BP}$, BP-OH, 2OH-BP, 3OH-BP, 4OH-BP, BP-1, BP-3, and BP-10 were prepared independently. Then, a mixture-standard solution $\left(10 \mu \mathrm{g} \mathrm{ml}^{-1}\right.$ ) was obtained by mixing the concentrated solutions. Urine samples were collected from fourteen healthy volunteers and sample preparation was performed immediately.

\section{Instrumentation}

A $10-\mu 1$ microsyringe for LPME was purchased from SGE Japan (Kanagawa, Japan). The microsyringe needle had a conical tip of $50 \mathrm{~mm}$ length and $0.63 \mathrm{~mm}$ o.d. An accurel Q 3/2 polypropylene hollow-fiber membrane of $600 \mu \mathrm{m}$ i.d., $200 \mu \mathrm{m}$ wall thickness, and a $0.2-\mu \mathrm{m}$ pore size was purchased from Membrana (Wuppertal, Germany). The hollow-fiber membrane was cut manually and carefully into $1.1 \mathrm{~cm}$ lengths. Then, the hollow-fiber segments were cleaned in acetone prior to use. For extraction, $2 \mathrm{ml}$ sample vials from Agilent Technologies (Palo
Alto, CA) were used.

\section{GC-MS instrument and analytical conditions}

GC-MS was performed with an Agilent 6890N gas chromatograph equipped with a $5973 \mathrm{~N}$ mass-selective detector (Agilent Technologies; Palo Alto, CA). Injection was performed in a pulsed splitless mode and the injection volume was $2 \mu \mathrm{l}$. The temperature of the inlet was $250^{\circ} \mathrm{C}$. Separation was conducted on a DB-5MS fused silica column $(30 \mathrm{~m} \times 0.25 \mathrm{~mm}$ i.d., $0.25 \mu \mathrm{m}$ film thickness, Agilent Technologies). The oven temperature was programmed to increase from $100^{\circ} \mathrm{C}$ (held for $1 \mathrm{~min}$ ) to $220^{\circ} \mathrm{C}$ at $5^{\circ} \mathrm{C} \mathrm{min}-1$, and then increased to $280^{\circ} \mathrm{C}$ (held

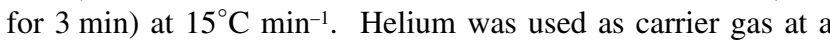
flow rate of $1.0 \mathrm{ml} \mathrm{min}{ }^{-1}$. The mass spectrometer was operated in the selected ion monitoring (SIM) mode with electron ionization (EI) (ionization voltage: $70 \mathrm{eV}$ ). The monitoring ions were as follows: $\mathrm{m} / \mathrm{z}, 182$ and 105 for BP, $\mathrm{m} / \mathrm{z}, \underline{213}$ and 256 for acyl-BP-1, $m / z, 227$ and 228 for acyl-BP-3, $m / z \underline{241}$ and 227 for acyl-BP-10, $\mathrm{m} / \mathrm{z} \underline{184}$ and 105 for BP-OH, $\mathrm{m} / z \underline{197}$ and 198 for acyl-2OH-BP, $m / z \underline{198}$ and 105 for acyl-3OH-BP, and $\mathrm{m} / \mathrm{z} \underline{121}$ and 198 for acyl-4OH-BP. The underlined number is the $m / z$ of the ion used for quantification. The monitoring ion for BP- $d_{10}$ was $m / z, 192$ as surrogate standard, and quantitative analyses of BPs were performed with a surrogate standard.

\section{Human urine sample preparation by LPME}

A human urine sample $(1 \mathrm{ml})$ spiked with surrogate standard was buffered with $1 \mathrm{M}$ ammonium acetate solution $(100 \mu \mathrm{l})$. After adding $\beta$-glucuronidase $\left(10 \mu \mathrm{l} ; 10000 \mathrm{U} \mathrm{ml}^{-1}\right)$ and sulfatase $\left(10 \mu \mathrm{l} ; 3650 \mathrm{U} \mathrm{ml}^{-1}\right)$, the sample was sealed in a glass tube and gently mixed. Enzymatic de-conjugation to release free BPs was performed by incubating at $37^{\circ} \mathrm{C}$ for $3 \mathrm{~h} .^{32}$ Then, a $300-\mu \mathrm{l}$ aliquot of the de-conjugated sample was transferred to another vial and mixed with the same volume of acetonitrile for deproteination. After shaking sufficiently, it was centrifuged for $10 \mathrm{~min}$ at $3000 \mathrm{rpm}$. A $500-\mu \mathrm{l}$ volume of supernatant was transferred to another vial and $1 \mathrm{ml}$ of purified water was added. Potassium carbonate $\left(1 \mathrm{M} \mathrm{K}_{2} \mathrm{CO}_{3} ; 50 \mu \mathrm{l}\right)$ for a $\mathrm{pH}$ adjustment and acetic acid anhydride $(20 \mu \mathrm{l})$ as a derivatization reagent were added, and the vial was degassed. Then, the sample was agitated. Finally, the sample was subjected to HF-LPME using a $10-\mu 1$ microsyringe. Before extraction, the microsyringe was rinsed 10 times each with acetone and toluene so as to avoid carryover and air-bubble formation. The needle tip was inserted into a 1.1-cm-long hollow fiber segment. Then, the fiber assembly was immersed in toluene for about $20 \mathrm{~s}$ to impregnate its pores with toluene. Toluene in the syringe was injected carefully into the hollow fiber, after which the fiber assembly was completely immersed in the sample solution. LPME was performed at room temperature for $15 \mathrm{~min}$ while stirring at $500 \mathrm{rpm}$. After extraction, $2 \mu \mathrm{l}$ of the extract was carefully withdrawn into the microsyringe and injected into the GC-MS system.

\section{Results and Discussion}

\section{Optimization of extraction solvent and time}

The extraction solvent was optimized. Toluene, butyl acetate, and 1-octanol were compared. When toluene was used as the extraction solvent, relatively high responses were obtained for all acyl-BPs. Therefore, toluene was used as the extraction solvent for the HF-LPME method.

One of the most important parameters affecting not only LPME, but also HF-LPME was the extraction time. To 


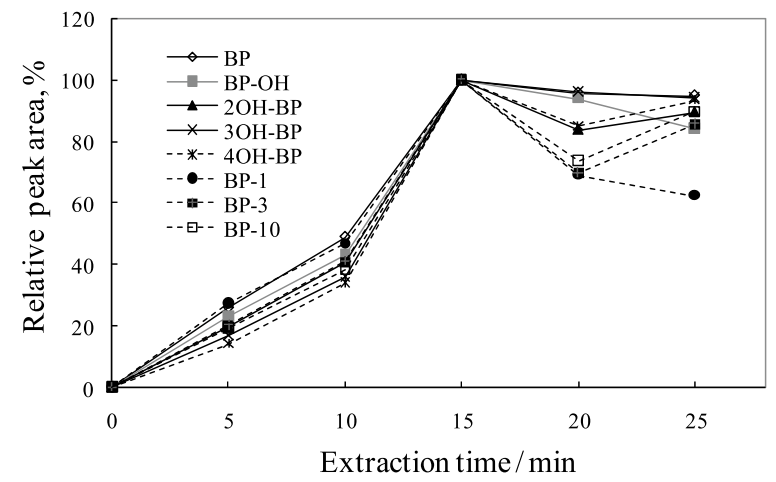

Fig. 1 Extraction time profiles of BPs. Optimum extraction time of analytes in $1 \mathrm{ml}$ standard solutions $\left(5 \mathrm{ng} \mathrm{ml}^{-1}\right)$ using HF-LPME with in situ derivatization and GC-MS.

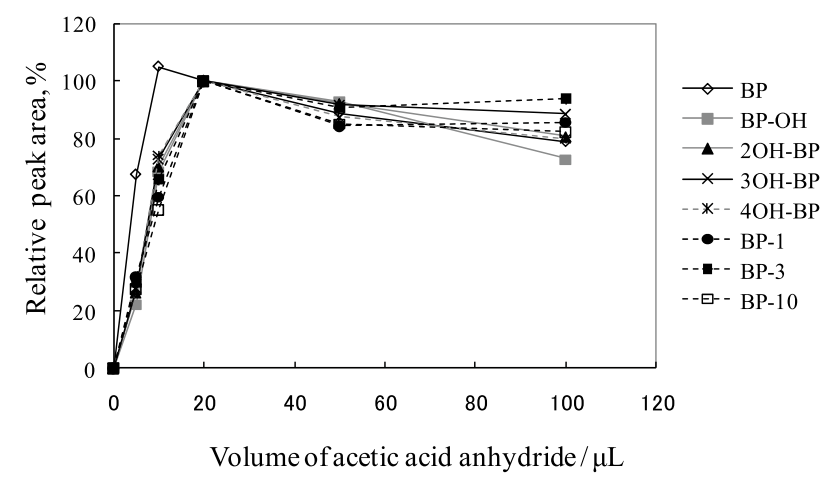

Fig. 2 Optimum volume of acetic acid anhydride for in situ derivatization. Optimum volume of acetic acid anhydride for in situ derivatization of BPs in $1 \mathrm{ml}$ standard solutions $\left(5 \mathrm{ng} \mathrm{ml}^{-1}\right)$ using HF-LPME with in situ derivatization and GC-MS.

determine the optimum extraction time, $5 \mathrm{ng} \mathrm{ml}^{-1}$ standard solutions of BPs were used. The extraction time profiles of $1 \mathrm{ml}$ standard solutions of the acyl derivatives of BPs using HF-LPME with in situ derivatization and GC-MS are shown in Fig. 1. The highest response was obtained when the extraction time was $15 \mathrm{~min}$. One possible reason for the decrease in the relative peak area was a reduction in the volume of toluene used as the extraction solvent. We thought that the extraction amounts of analytes were decreased according to the decreasing volume of the extraction solvent. This condition (15 min) was therefore used for the determination of BPs in human urine samples.

\section{Optimization of in situ derivatization and GC-MS conditions}

The volumes of $\mathrm{K}_{2} \mathrm{CO}_{3}$ (5 to $\left.500 \mu \mathrm{l}\right)$ and acetic acid anhydride ( 0 to $50 \mu \mathrm{l})$ in the in situ derivatization step were optimized. When $50 \mu \mathrm{l}$ of $\mathrm{K}_{2} \mathrm{CO}_{3}$ was used for a $\mathrm{pH}$ adjustment, all BPs showed relatively high responses. As shown in Fig. 2, the highest response was obtained from BP when $10 \mu \mathrm{l}$ of acetic acid anhydride was used. When $20 \mu \mathrm{l}$ of acetic acid anhydride was used for the in situ derivatization of BPs, relatively high responses were obtained as well (Fig. 2). Therefore, $20 \mu \mathrm{l}$ of acetic acid anhydride was used as the optimum volume. We thought that the $\mathrm{pH}$ changed when it was added over the $20 \mu \mathrm{l}$ of acetic acid anhydride.

An EI-MS analysis of the standard solutions of analytes in the

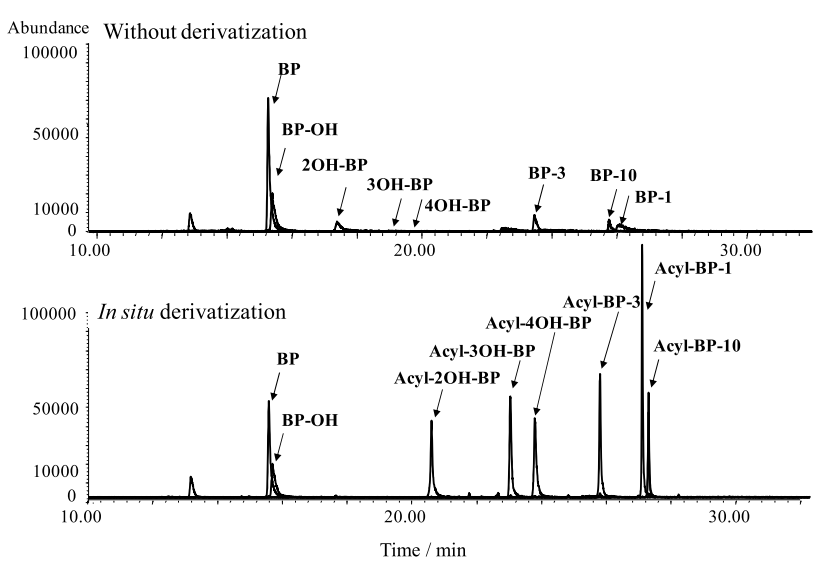

Fig. 3 Comparison of chromatograms of BPs obtained with and without derivatization. For HF-LPME with in situ derivatization, derivatization reagents were added to $1 \mathrm{ml}$ of BP standard solution (500 $\mathrm{ng} \mathrm{ml}^{-1}$ ) and the extraction was commenced for $15 \mathrm{~min}$ at room temperature in a glass vial. Then, the extraction solvent was subjected to GC-MS analysis. For HF-LPME without in situ derivatization, the same procedure was performed, except that no derivatization reagents were added.

Table 1 Analytical figures of merit of HF-LPME with in situ derivatization and GC-MS

\begin{tabular}{lcccc}
\hline & $\begin{array}{c}\text { LOD/ } \\
\mathrm{ng} \mathrm{m}^{-1}\end{array}$ & $\begin{array}{c}\text { LOQ/ } \\
\mathrm{ng} \mathrm{ml}^{-1}\end{array}$ & $\begin{array}{c}\text { Linear range/ } \\
\mathrm{ng} \mathrm{ml}^{-1}\end{array}$ & $\begin{array}{c}\text { Correlation } \\
\text { coefficient, } \\
r\end{array}$ \\
\hline BP & 0.02 & 0.1 & $0.1-100$ & 0.999 \\
BP-OH & 0.05 & 0.2 & $0.2-100$ & 0.999 \\
2OH-BP & 0.02 & 0.1 & $0.1-100$ & 0.995 \\
3OH-BP & 0.02 & 0.1 & $0.1-100$ & 0.998 \\
4OH-BP & 0.02 & 0.1 & $0.1-100$ & 0.997 \\
BP-1 & 0.02 & 0.1 & $0.1-100$ & 0.998 \\
BP-3 & 0.01 & 0.05 & $0.05-100$ & 0.996 \\
BP-10 & 0.02 & 0.1 & $0.1-100$ & 0.996 \\
\hline
\end{tabular}

LOD, Limit of detection $(S / N=3)$; LOQ, limit of quantification $(S / N>10)$.

scan mode was conducted. Major and minor signals were used as quantification and qualifier ions, respectively. The monitoring ions were as follows: $\mathrm{m} / \mathrm{z} \underline{182}$ and 105 for BP, $\mathrm{m} / \mathrm{z}, \underline{213}$ and 256 for acyl-BP-1, $m / z, 227$ and 228 for acyl-BP-3, $m / z \underline{241}$ and 227 for acyl-BP-10, $m / z \underline{184}$ and 105 for BP-OH, $\mathrm{m} / z \underline{197}$ and 198 for acyl-2OH-BP, $m / z, 198$ and 105 for acyl-3OH-BP, and $\mathrm{m} / \mathrm{z}$ 121 and 198 for acyl-4OH-BP.

The effect of in situ derivatization was examined. As shown in Fig. 3, peaks of 3OH-BP and 4OH-BP could be detected in the case with in situ derivatization. Moreover, the peaks of other acyl-BPs became sharp, since the phenolic hydroxyl group was derivatized. Therefore, in situ derivatization was a useful method for the determination of trace levels of BPs in human urine samples.

\section{Analytical figures of merit}

The limits of detection (LODs) (signal-noise ratio: $S / N=3$ ) and the limits of quantification (LOQs) $(S / N>10)$ of BPs in human urine samples subjected to in situ derivatization were 0.01 to 0.05 and 0.05 to $0.2 \mathrm{ng} \mathrm{ml}^{-1}$, respectively. For BP 

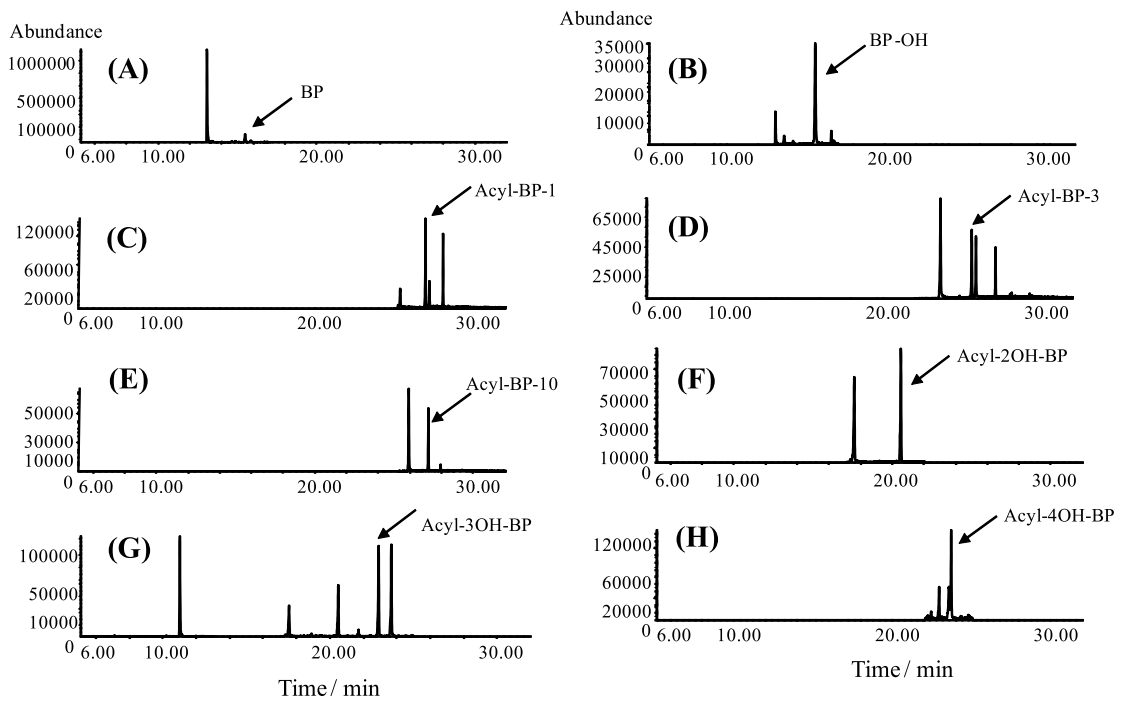

Fig. 4 Typical chromatograms of spiked urine samples. SIM chromatograms $\left(50 \mathrm{ng} \mathrm{ml}^{-1}\right.$ spiked in urine sample) of BP (A), BP-OH (B), acyl-BP-1 (C), acyl-BP-3 (D), acyl-BP-10 (E), acyl-2OH-BP (F), acyl-3OH-BP (G), and acyl-4OH-BP (H) were monitored at $m / z, 182,184,213,227,241,197,198$, and 121 , respectively.

Table 2 Recoveries of BPs in human urine samples

\begin{tabular}{lcccccc}
\hline & \multicolumn{2}{c}{$10 \mathrm{ng} \mathrm{ml}^{-1}$ spiked } & & \multicolumn{2}{c}{$50 \mathrm{ng} \mathrm{ml}^{-1}$ spiked } \\
\cline { 2 - 3 } \cline { 5 - 6 } & Recovery, \% & RSD, \% & & Recovery, \% & RSD, \% \\
\hline BP & 101.5 & 5.8 & & 99.5 & 5.7 \\
BP-OH & 97.5 & 6.0 & & 99.7 & 3.4 \\
2OH-BP & 97.9 & 1.6 & & 100.8 & 3.0 \\
3OH-BP & 97.9 & 1.5 & & 100.8 & 3.9 \\
4OH-BP & 97.8 & 2.1 & & 100.2 & 4.2 \\
BP-1 & 106.7 & 7.7 & & 101.5 & 3.4 \\
BP-3 & 93.1 & 8.4 & & 99.5 & 4.6 \\
BP-10 & 95.8 & 7.6 & & 96.3 & 7.7 \\
\hline
\end{tabular}

The recoveries and RSD were also examined by replicate analysis $(n=5)$ of human urine samples.

determination, calibration curves were obtained by plotting the peak-area ratio versus the concentration. The calibration curves for analytes were linear with correlation coefficients $>0.995$ in the range of 0.05 to $100 \mathrm{ng} \mathrm{ml}^{-1}$ for BP-3, 0.2 to $100 \mathrm{ng} \mathrm{ml}^{-1}$ for $\mathrm{BP}-\mathrm{OH}$, and 0.1 to $100 \mathrm{ng} \mathrm{ml}^{-1}$ for the other BPs (Table 1). The relative recovery and precision of the method were assessed by replicate analyses $(n=5)$ of human urine samples spiked at 10 and $50 \mathrm{ng} \mathrm{ml}^{-1}$. Non-spiked and spiked samples were subjected to HF-LPME with in situ derivatization and GC-MS. Typical chromatograms of spiked urine samples are shown in Fig. 4. The relative recoveries were calculated by subtracting the results for non-spiked samples from those for spiked samples. The results were obtained by using calibration curves of the standard solutions with surrogate standards. The average recoveries of analytes $(n=5)$ in human urine samples spiked with 10 and $50 \mathrm{ng} \mathrm{ml}^{-1}$ BPs were 93.1 to $106.7 \%$ (RSD: 1.5 to $8.4 \%$ ) and 96.3 to $101.5 \%$ (RSD: 3.0 to $7.7 \%$ ), respectively (Table 2). Therefore, this method enables precise determinations of standards, and can be applied to the determination of BPs in human urine samples.
Table 3 Concentrations of BPs in human urine samples

\begin{tabular}{ccccccccc}
\hline & \multicolumn{7}{c}{ Concentration of BPs/ng ml-1 } \\
\cline { 2 - 8 } Volunteer & BP & $\begin{array}{c}\text { BP- } \\
\text { OH }\end{array}$ & $\begin{array}{c}\text { 2OH- } \\
\text { BP }\end{array}$ & $\begin{array}{c}\text { 3OH- } \\
\text { BP }\end{array}$ & $\begin{array}{c}\text { 4OH- } \\
\text { BP }\end{array}$ & BP-1 & BP-3 & BP-10 \\
\hline A & N.D. & 0.27 & N.D. & N.D. & N.D. & 0.32 & 1.23 & N.D. \\
B & N.D. & 0.70 & N.D. & N.D. & N.D. & N.D. & 0.73 & N.D. \\
C & N.D. & 2.48 & N.D. & N.D. & N.D. & 0.54 & 1.56 & N.D. \\
D & N.D. & 5.16 & N.D. & N.D. & N.D. & N.D. & 2.59 & N.D. \\
E & N.D. & 6.36 & N.D. & N.D. & N.D. & N.D. & 1.02 & N.D. \\
F & N.D. & 4.10 & N.D. & N.D. & N.D. & N.D. & 0.55 & 0.56 \\
G & N.D. & 10.0 & N.D. & N.D. & N.D. & N.D. & 0.48 & 0.71 \\
H & N.D. & 2.65 & N.D. & N.D. & N.D. & N.D. & 0.45 & N.D. \\
I & N.D. & 1.37 & N.D. & N.D. & N.D. & 1.15 & 4.29 & 0.94 \\
J & N.D. & 0.78 & N.D. & N.D. & N.D. & 0.75 & 4.91 & N.D. \\
K & N.D. & 0.70 & N.D. & N.D. & N.D. & N.D. & 0.36 & N.D. \\
L & N.D. & 0.94 & N.D. & N.D. & N.D. & 1.91 & 1.67 & 1.07 \\
M & N.D. & 2.11 & N.D. & N.D. & 0.15 & 3.13 & 6.91 & 1.31 \\
N & N.D. & 4.14 & N.D. & N.D. & N.D. & N.D. & 2.25 & N.D. \\
\hline
\end{tabular}

N.D. indicates not determined.

\section{Determination of BPs in human urine samples}

Urine samples from fourteen healthy volunteers (five females and nine males) were analyzed using the present method. BP, $2 \mathrm{OH}-\mathrm{BP}$ and $3 \mathrm{OH}-\mathrm{BP}$ were not detected in the human urine samples. In contrast, BP-OH and BP-3 were detected in all urine samples in the range of 0.27 to $10.0 \mathrm{ng} \mathrm{ml}^{-1}$ and 0.36 to $6.91 \mathrm{ng} \mathrm{ml}^{-1}$, respectively (Table 3 ). Trace amounts of BP-1, BP-10, and 4OH-BP were detected in some samples.

The Ministry of the Environment has published an annual report, "Chemicals in the Environment, FY2005," which contains the results of an environmental survey and monitoring of chemicals. The Ministry of the Environment has also conducted indoor-air monitoring to determine BPs. ${ }^{33}$ The report states that BP and BP-3 were determined frequently in the 68 indoor air samples analyzed. Actually, BP and BP-3 were detected in 67 indoor air samples. Taking the frequent detection 
of BP-3 into consideration, it was reasonable that BP-3 was detected in all human urine samples. Meanwhile, BP, which was also detected in indoor air samples, was metabolized to $\mathrm{BP}-\mathrm{OH}$, which was detected in all human urine samples.

The combination of HF-LPME with in situ derivatization and GC-MS led to the successful determination of trace amounts of BPs in human urine samples. We have previously reported on the analysis of BPs in a human urine sample by SBSE-TD-GCMS. ${ }^{18}$ In order to improve the sensitivity and cost performance, we also developed miniaturized HF-LPME for BP analysis. ${ }^{32}$ In that study, ${ }^{32}$ five kinds of BPs (BP, BP-OH, 2OH-BP, BP-3, and BP-10) could be analyzed without derivatization. However, an even wider variety of BP-related compounds should be analyzed to reveal the extent of exposure to BPs. Using our proposed improvement method with in situ derivatization, BP-1, 3OH-BP, and $4 \mathrm{OH}-\mathrm{BP}$ were detected, although they could not be detected when in situ derivatization was not performed. Moreover, previous GC conditions should be modified to improve the separation of BPs.

This work is the first to determine trace amounts of BPs in human urine samples using HF-LPME with in situ derivatization and GC-MS. The proposed method has many practical advantages, including simplicity of the extraction method, the use of a small volume of organic solvent for extraction, and high sensitivity, and is sufficiently applicable to analyses of human urine samples. The proposed method is expected to have potential applications in human urine samples.

\section{Acknowledgements}

This study was supported by Health Sciences Research grants from the Ministry of Health, Labour and Welfare of Japan.

\section{References}

1. F. J. Moloney, S. Collins, and G. M. Murphy, Am. J. Clin. Dermatol., 2002, 3, 185.

2. J. L. Bourrain, P. Amblard, and J. C. Beani, Contact Dermatitis, 2003, 48, 45.

3. M. Schlumpf, B. Cotton, M. Conscience, V. Haller, B. Steinmann, and W. Lichtensteiger, Environ. Health Perspect., 2001, 109, 239.

4. Y. Nakagawa and T. Suzuki, Chem. Biol. Interact., 2002, 156, 27.

5. S. Takatori, Y. Kitagawa, H. Oda, G. Miwa, J. Nishikawa, T. Nishihara, H. Nakagawa, and S. Hori, J. Health Sci., 2003, 49, 91 .

6. C. Nerín and E. Asensio, Anal. Bioanal. Chem., 2007, 389, 589.

7. L. Castle, C. P. Offen, M. J. Baxter, and J. Gilbert, Food Addit. Contam., 1997, 14, 35.

8. Y. Nakagawa, T. Suzuki, and S. Tayama, Toxicology, 2000
156,27

9. V. Sarveiya, S. Risk, and H. A. E. Benson, J. Chromatogr., $B, \mathbf{2 0 0 4}, 803,225$.

10. X. Ye, Z. Kuklenyik, L. L. Needham, and A. M. Calafat, Anal. Chem., 2005, 77, 5407.

11. X. Ye, Z. Kuklenyik, L. L. Needham, and A. M. Calafat, Anal. Bioanal. Chem., 2005, 383, 638.

12. T. Felix, B. J. Hall, and J. S. Brodbelt, Anal. Chim. Acta, 1998, 371, 195.

13. L. H. Wang, Chromatographia, 1999, 50, 565.

14. H.-K. Jeon, Y. Chung, and J.-C. Ryu, J. Chromatogr., A, 2006, 1131, 192.

15. A. L. Gregory and E. P. Mark, Environ. Sci. Technol., 2006, 40, 687.

16. M. Kawaguchi, R. Ito, N. Endo, N. Sakui, N. Okanouchi, K. Saito, N. Sato, T. Shiozaki, and H. Nakazawa, Anal. Chim. Acta, 2006, 557, 272.

17. M. Kawaguchi, R. Ito, H. Honda, N. Endo, N. Okanouchi, K. Saito, Y. Seto, and H. Nakazawa, J. Chromatogr., A, 2008, 1200, 260 .

18. M. Kawaguchi, R. Ito, H. Honda, N. Endo, N. Okanouchi, K. Saito, Y. Seto, and H. Nakazawa, Anal. Sci., 2008, 24, 1509.

19. M. A. Jeannot and F. F. Cantwell, Anal. Chem., 1996, 68, 2236.

20. Y. He and H. K. Lee, Anal. Chem., 1997, 69, 4634.

21. M. A. Jeannot and F. F. Cantwell, Anal. Chem., 1997, 69, 235.

22. L. Zhao and H. K. Lee, Anal. Chem., 2002, 74, 2486.

23. J.-F. Liu, G.-B. Jiang, Y.-G. Chi, Y.-Q. Cai, Q.-X. Zhou, and J.-T. Hu, Anal. Chem., 2003, 75, 5870.

24. C. Basheer, R. Balasubramanian, and H. K. Lee, J. Chromatogr., A, 2003, 1016, 11.

25. C. Basheer, H. K. Lee, and J. P. Obbard, J. Chromatogr., A, 2004, 1022, 161 .

26. C. Basheer, J. P. Obbard, and H. K. Lee, J. Chromatogr., A, 2005, 1068, 221.

27. L. Hou and H. K. Lee, J. Chromatogr., A, 2004, 1038, 37.

28. C.-H. Yan and H.-F. Wu, Rapid Commun. Mass Spectrom., 2004, 18, 3015.

29. L. Hou, G. Shen, and H. K. Lee, J. Chromatogr., A, 2003, 985, 107.

30. V. Colombini, C. Bancon-Montigny, L. Yang, P. Maxwell, R. E. Sturgeon, and Z. Mester, Talanta, 2004, 63, 555.

31. R. Ito, M. Kawaguchi, H. Honda, Y. Koganei, N. Okanouchi, N. Sakui, K. Saito, and H. Nakazawa, J. Chromatogr., B, 2008, 872, 63 .

32. M. Kawaguchi, R. Ito, H. Honda, N. Okanouchi, K. Saito, Y. Seto, and H. Nakazawa, J. Chromatogr., B, 2009, 877, 298.

33. The Ministry of the Environment (MOE), 2006, http://www.env.go.jp/chemi/kurohon/2005/http2005/ 30furoku/313.pdf (in Japanese). 